\title{
Distortion of magnetic fields in the pre-stellar core Barnard 68
}

\author{
Ryo Kandori, Motohide Tamura, Ken-ichi Tatematsu, Nobuhiko \\ Kusakabe, Yasushi Nakajima, and the IRSF/SIRPOL group
}

\author{
National Astronomical Observatory, 2-21-1 Osawa, Mitaka, Tokyo 181-8588 \\ email: r.kandori@nao.ac.jp
}

\begin{abstract}
Magnetic fields are believed to play an important role in controlling the stability and contraction of molecular cloud cores. In the present study, magnetic fields of a cold pre-stellar core, Barnard 68, have been mapped based on wide-field near-infrared polarimetric observations of background stars. A distinct "hourglass-shaped" magnetic field is identified toward the core, as the observational evidence of magnetic field structure distorted by mass accumulation in a pre-stellar core. Our findings on the geometry of magnetic fields as well as the mass-to-magnetic flux ratio are presented.
\end{abstract}

Keywords. Stars: formation - ISM: globules - ISM: magnetic fields - polarization

\section{Introduction}

The characteristics of newborn stars are thought to be determined primarily by the properties of the molecular cloud core prior to the onset of gravitational collapse, and the magnetic field pervading the core is believed to play an important role in controlling the stability and contraction of the pre-stellar core (e.g., Shu, Adams, \& Lizano 1987). It is therefore of importance to investigate the magnetic fields affecting the cores in the pre-stellar phase to clarify the initial conditions of star formation. The direction of magnetic fields projected onto the sky can be inferred from polarimetric observations of dust emission ( $\mathrm{B} \perp \mathrm{E}$ ) and/or dichroic extinction (B \| E, e.g., Lazarian et al. 2007). Dust emission polarimetry, particularly at sub-millimeter to far-infrared wavelengths, has proven to be a powerful technique for tracing magnetic field structures in dense regions, such as in protostellar envelopes (e.g., Girart et al. 2006). However, polarized dust emission from cold pre-stellar cores is too weak to be detected by present instruments except for dense central regions (e.g., Ward-Thompson, 2000). There are thus no observations of pre-stellar cores tracing magnetic fields from the center to the outermost regions.

\section{Magnetic Properties of Barnard 68}

In the present study, a magnetic field map of the pre-stellar core Barnard 68 (hereafter B68) is constructed on the basis of near-infrared polarimetry toward background stars suffering dichroic extinction by magnetically aligned dust in the core. Observations were made in 2007 June using the near-infrared imaging polarimeter SIRPOL (Kandori et al. 2006) on the IRSF 1.4-m telescope in South Africa. IRSF/SIRPOL provides deep (18.6 mag in the $H$ band, $5 \sigma$ in one hour exposure) and wide-field $\left(7.7^{\prime} \times 7.7^{\prime}\right)$ polarization images. In Figure 1, the $H$ band polarization vectors of 125 background stars (yellow lines) toward B68 are shown. The white lines show the magnetic field configuration inferred from the fitting using a parabolic function. The fitting appears reasonable, since the rms of residual angles of polarization vectors in the fitting is smaller for the parabolic 

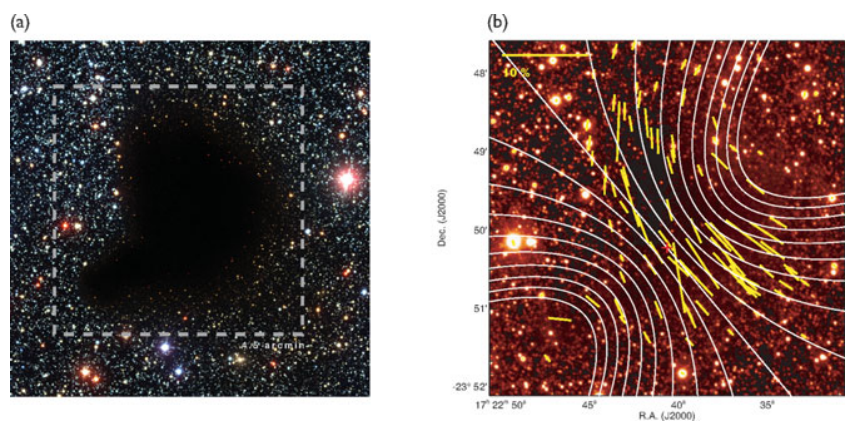

Figure 1. (a) - Optical image of B68 (Alves et al. 2001). (b) - $H$ band polarization vectors of 125 background stars (yellow lines) on the intensity image. The white lines show the most probable magnetic field configuration inferred from the fitting using a parabolic function, $y=g+g C x^{2}$, with the magnetic axis position angle of $\sim 40^{\circ}$ and $C \sim 2 \times 10^{-4} \operatorname{arcsec}^{-2}$.

field case than for the linear case. The magnetic field seems to follow a distinct not linear axi-symmetric shape reminiscent of an hourglass. The orientation of the magnetic axis $\left(\sim 40^{\circ}\right)$ of $\mathrm{B} 68$ is nearly perpendicular to the major axis of the core elongation $\left(\sim 130^{\circ}\right)$ in the dense regions $\left(A_{V}>10 \mathrm{mag}\right)$. These geometrical magnetic properties are consistent with theoretical suggestions that (1) initially straight frozen-in magnetic fields pervading a core can be dragged toward the center by the contracting medium and distorted into an hourglass-shaped structure, and (2) a disk-like structure can eventually be formed in the center by mass accretion along the magnetic field lines, with the major axis of the disk aligned perpendicular to the magnetic axis (e.g., Galli \& Shu 1993).

Given the magnetic field structure and other properties known for B68 (e.g., Alves et al. 2001), the strength of the plane-of-sky magnetic field was obtained as $20 \pm 5 \mu \mathrm{G}$ based on the Chandrasekhar-Fermi method (Chandrasekhar \& Fermi, 1953) with a theoretically suggested correction factor of 0.5 (Ostriker et al. 2001). The comparison of the observed mass-to-magnetic flux to a critical value suggested by theory (Nakano \& Nakamura 1978) resulted in $(M / \Phi)_{\text {obs }} /(M / \Phi)_{\text {critical }} \sim 2.3$ (magnetically supercritical). The result does not necessarily mean the gravitational collapse, because B68 could be marginally stable from the support by thermal and (small) turbulent pressures (e.g., Lada et al. 2003). The magnetic fields can provide an extra support to retain the core to be stable. Finally we speculate the origin of hourglass field structure. Since B68 seems stable in the present phase, the structure is interpreted as an imprint of mass condensation during the development of the core under the influence of the gravity and turbulence.

\section{References}

Alves, J. F., Lada, C. J., \& Lada, E. A. 2001, Nature 409, 159

Chandrasekhar, S. \& Fermi, E. 1953, ApJ 118, 113

Galli, D. \& Shu, F. 1993, ApJ 417, 220

Girart, J. M., Rao, R., \& Marrone, D. P. 2006, Science 313, 812

Kandori, R., et al. 2006, Proc. SPIE 6269, 159

Lada, C. J., Bergin, E. A., Alves, J. F., \& Huard, T. L. 2003, ApJ 586, 286

Lazarian, A. 2007, J. Quant. Spectrosc. Rad. Trans. 106, 225

Nakano, T. \& Nakamura, T. 1978, PASJ 30, 671

Ostriker, E. C., Stone, J. M., \& Gammie, C. F. 2001, ApJ 546, 980

Shu, F., Adams, F. C., \& Lizano, S. 1987, ARA\&A 25, 23

Ward-Thompson, D., et al. 2000, ApJ 537, 135 